\title{
Physico-Chemical and Functional Properties of Traditional Sorghum Landraces
}

\author{
Preeti Ramakant Hungund $^{1 *}$, Usha Malagi ${ }^{1}$ and N. G. Hanamaratti ${ }^{2}$ \\ ${ }^{1}$ Department of Food Science and Nutrition, UAS, Dharwad, Karnataka, India \\ ${ }^{2}$ AICRP on Sorghum, College of Community Science, UAS, Dharwad, Karnataka, India \\ *Corresponding author
}

\section{A B S T R A C T}

\section{Keywords}

Sorghum, landraces, physico-chemical properties and functional properties

\section{Article Info}

Accepted:

18 March 2021

Available Online:

10 April 2021
The study of physico-chemical properties of grains is a basic step in any research and it is important to maintain end product quality and to determine consumer acceptability. Evaluation of nutritional values of different sorghum genotypes help sorghum breeders for improvement of varieties based on nutritional aspects. The present study was undertaken to compare physico-chemical properties of twenty sorghum landraces with high yielding variety (M35-1). The physical properties viz., 100 kernel weight, density, colour values, of most of the traditional sorghum landraces were significantly higher compared to M35-1. The functional properties of landraces were on par with M35-1. In proximate composition moisture, fat and carbohydrate content of landraces were on par with M35-1. The crude protein and crude fiber content were significantly higher and the ash content of most of the landraces was significantly lower compared to M351.

\section{Introduction}

Sorghum is the fifth most important cereal crop after wheat, rice, maize, barley and is the dietary staple of more than 500 million people in more than 30 countries. It is important food crop for economical weaker sections and for traditional consumers because of its two fold importance i.e., utilization of sorghum fodder and stover as feed for animals and that of grain as human food. Sorghum also has the potential for grain production even under low rainfall and it sustains adverse agro-climatic conditions. Compounds like 3Deoxyanthoxyanins (3-DXA) are present in darker-coloured sorghums and to a lesser extent in white sorghum and it has strong antiproliferative activity against human colon 
cancer cells (Yang et al., 2009). Sorghum being gluten-free cereal attracts attention of researchers in the present day scenario where the occurrence of Celiac Disease (CD), an immunological response to gluten intolerance is on the rise. Sorghum landraces are basic materials to develop improved varieties.

These traditional sorghum landraces have unique source of specific traits for disease and pest resistance, nutritional quality, tolerance to marginal environment and adverse climatic condition.

For subsequent inclusion of landraces in breading programme a detail research on physico-chemical properties of traditional sorghum landraces is necessary. Hence, the objective of present study is to compare physico-chemical properties of traditional sorghum landraces with high yielding variety M35-1.

\section{Materials and Methods}

\section{Sample procurement}

The experiment was conducted during the year 2019-2020 at the department of Food Science and Nutrition, College of Community Science, UAS, Dharwad. A total twenty sorghum landraces and one high yielding variety M35-1 was procured from AICRP Sorghum, UAS, Dharwad.

\section{Sorghum grain properties}

The traditional sorghum landraces were analysed for physical properties viz., kernel weight, volume and density.

\section{0 kernel weight}

Weight of randomly selected hundred grains in grams was recorded using electronic balance with a sensitivity of $0.1 \mathrm{mg}$.

\section{0 kernel volume}

Hundred randomly selected grains were dropped in measuring cylinder containing known volume of distilled water. Increase in volume of water was noted and the difference was recorded in $\mathrm{ml}$ as volume.

\section{Density}

Bulk density of seed was calculated using the formula:

Bulk density $(\mathrm{g} / \mathrm{ml})$

Seed weight $(\mathrm{g})$

Seed volume (ml)

\section{Colour}

Twenty sorghum landraces and one high yielding sorghum variety (M35-1) were ground to flour and subjected to colour assessment in spectrophotometer Konica Minolta. The colour was measured in chromatic components of $\mathrm{L}^{*}, \mathrm{a}^{*}$ and $\mathrm{b}^{*}$ values. ' $L$ ' value measures black (0) to white (100), ' $a$ ' represents redness ( + a values) to greenness $(-a$ values) and ' $b$ ' represents yellowness $(+b$ values) to blueness (-b values).

\section{Functional properties of sorghum flour}

\section{Water absorption capacity}

Water absorption capacity was assessed by the method of Quin and Paton (1983). Five gram of flour was weighed and added to preweighed centrifuge tube $\left(\mathrm{W}_{1}\right)$. To this, $30 \mathrm{ml}$ of water was added and stirred with a glass rod for five minutes. The contents were allowed to stand for 30 minutes and then centrifuged at 11,000 rpm for 25 minutes. The free liquid was poured off. Inner side of tube were wiped with tissue paper. The centrifuge tube was weighed again $\left(\mathrm{W}_{2}\right)$. The water 
absorption capacity was calculated using formula.

Water absorption capacity, WAC (g/g)

$$
=\frac{\mathrm{W}_{2}-\mathrm{W}_{1}}{5} \times 100
$$

Where, $\mathrm{W}_{1}=$ weight of sample plus centrifuge tube

$\mathrm{W}_{2}=$ weight of sample plus centrifuge tube after centrifuge

\section{Oil Absorption Capacity}

Oil absorption capacity was measured using the method given by Sosulski et al., (1976). One gram of flour was mixed with $10 \mathrm{ml}$ of vegetable oil (groundnut oil) in weighed centrifuge tube $\left(\mathrm{W}_{1}\right)$. The tubes were stirred for one minute for complete dispersion of sample in the oil. After $30 \mathrm{~min}$ of holding time at room temperature, the sample was centrifuged at $3000 \mathrm{rpm}$ for $25 \mathrm{~min}$. The separated oil was then removed and tubes were inverted on oil absorbent paper to drain the oil prior to reweighing $\left(\mathrm{W}_{2}\right)$. The oil absorption capacity was calculated using the formula.

Oil absorption capacity, OAC (g/g)

$$
=\frac{\mathrm{W}_{2}-\mathrm{W}_{1}}{1} \times 100
$$

Where, $\mathrm{W}_{1}=$ Weight of sample plus centrifuge tube

$\mathrm{W}_{2}=$ Weight of sample plus centrifuge tube after centrifuging and discarding supernatant

\section{Swelling power and per cent solubility}

The swelling power and per cent solubility were determined according to the method used by Schoch (1964). Five hundred milligram $\left(\mathrm{W}_{1}\right)$ of sample was added to a centrifuge tube, weight of centrifuge tube with sample was noted $\left(\mathrm{W}_{2}\right)$. After addition of $20 \mathrm{ml}\left(\mathrm{V}_{\mathrm{E}}\right)$ distilled water, the centrifuge tube was placed in the water bath at $100{ }^{\circ} \mathrm{C}$ for 30 min till the contents were cooked. Then it was centrifuged at $5000 \mathrm{rpm}$ for $10 \mathrm{~min}$. The supernatant was transferred to a test tube and the inner side of the centrifuge tube was dried well and weighed $\left(\mathrm{W}_{3}\right)$. The swelling of flour was calculated as follows.

Swelling power $(\mathrm{g} / \mathrm{g})$

$$
=\frac{\mathrm{W}_{3}-\mathrm{W}_{2}}{\mathrm{~W}_{1}}
$$

Where, $\mathrm{W}_{1}=$ Weight of food samples

$\mathrm{W}_{2}=$ Weight of the centrifuge tube with food sample

$\mathrm{W}_{3}=$ Weight of the centrifuge tube after centrifuge

For per cent solubility, weight of dried petri dish was noted $\left(\mathrm{W}_{4}\right), 10 \mathrm{ml}$ aliquot $\left(\mathrm{V}_{\mathrm{A}}\right)$ was poured in the dish and dried at $110{ }^{\circ} \mathrm{C}$ for $4-5$ hr or until dry. The moisture dish was cooled in desiccator and weighed $\left(\mathrm{W}_{5}\right)$.

Solubility (\%)

$\left(\mathrm{W}_{5}-\mathrm{W}_{4}\right) \mathrm{V}_{\mathrm{E}} 100$

$\mathrm{V}_{\mathrm{A}} \quad \mathrm{W}_{1}$

Where, $\mathrm{W}_{1}=$ Weight of the sample

$\mathrm{W}_{4}=$ Weight of the petriplate

$\mathrm{W}_{5}=$ Weight of the petriplate with dry solids

$\mathrm{V}_{\mathrm{E}}=$ Volume of water added

$\mathrm{V}_{\mathrm{A}}=$ Volume of supernatant 


\section{Proximate composition of sorghum landraces \\ Moisture \\ Moisture content of the sample was expressed in per cent (Anon., 2005).

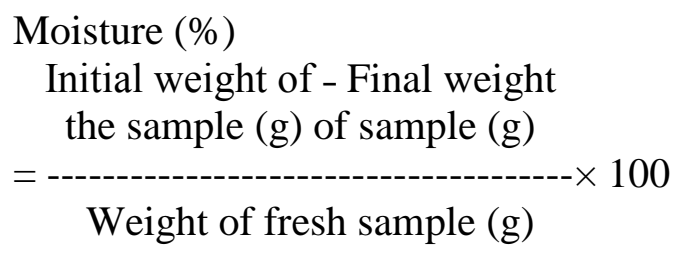

\section{Fat}

Soxhlet apparatus was used to determined crude fat content of the samples. The percent of crude fat was expressed as follows.

(\%) Crude fat

$$
\begin{aligned}
& \text { Final weight- Empty weight } \\
& \text { of flask of flask } \\
& \text { Weight of sample }
\end{aligned}
$$

\section{Protein}

Protein content was determined using Anon., 2005 method. Per cent nitrogen and protein was calculated by the following equation.

Nitrogen $(\%)$

$14 \times$ normality of the acid $x$ (titrant value burette reading) = -

Sample weight $\times 1000$

Protein $(\%)=\% \mathrm{~N} \times 6.25$

\section{Ash}

Ash was determined using muffle furnace (Anon., 2005). The percent ash was calculated using following formula.
Ash content (g \%)

Weight of the ash

= - - 100

Weight of the sample

\section{Crude fiber}

The crude fiber content of sorghum determined by Anon., 2005 method.

(\%) Crude fiber

Weight of residue - Weight of ash

$$
\text { Weight of sample }
$$

\section{Total carbohydrate}

Total carbohydrate was determined by difference that is by subtracting the measured protein, fat, ash and moisture from 100.

\section{Statistical analysis}

The SPSS version of 16.0 package was used to carry out statistical analysis. One way ANOVA was applied to study variation among twenty traditional sorghum landraces. Dunnet's test was applied to compare physicochemical and functional properties of sorghum landraces with high yielding variety.

\section{Results and Discussion}

The physical properties of sorghum genotypes are presented in table 1 . There was significant variation in 100 kernel weight, 100 kernel volume and density $(p \leq 0.001)$ among the sorghum landraces. The 100 kernal weight ranged from 2.31 to $4.23 \mathrm{~g}, 100$ kernel volume ranged from 2.00 to $3.67 \mathrm{ml}$, bulk density ranged from 0.83 to $1.42 \mathrm{~g} / \mathrm{ml}$. Most of the landraces showed significantly higher 100 kernel weight and bulk density when compared with M35-1. The three landraces viz., Annigeri-1 $(\mathrm{P}=0.000)$, Lakamapur-local $(\mathrm{P}=0.000)$ and SBABM jola $(\mathrm{P}=0.047)$ had 
significantly higher 100 kernel volume, while five landraces viz., Basavanapada $(\mathrm{P}=0.000)$, Giddamaldandi ( $\mathrm{P}=0.013)$, Nilugal $(\mathrm{P}=0.047)$, Rekkada jola $(\mathrm{P}=0.013)$ and Yenigararti $(\mathrm{P}=0.000)$ had significantly lower 100 kernel volume compared to M35-1. The other landraces were on par with M35-1. This variation of physical properties among sorghum landraces can be attributed to differences in endosperm colour, texture (corneous and floury) and endosperm type (normal, waxy or sugary). Patekar et al., 2017, reported lower 100 kernel weight, 100 kernel volume and density of five sorghum hybrids compared to studied traditional sorghum landraces.

Itagi and Hemalatha, 2017, found rabi sorghum landraces had significantly higher 100 kernel weight, volume and density than released/advanced rabi sorghum genotypes. These differences in physical properties of sorghum in the present study compared to other investigations may be due to varietal, location and climatic differences. The colour values $\left(L^{*}, a^{*}\right.$ and $\left.b^{*}\right)$ of traditional sorghum landraces was darker and redder compared to M35-1. The exposure of sorghum grains to moisture before and after harvesting might have caused pigmented pericarp.

Functional properties of sorghum landraces are presented in table 2 . There was significant variation $(\mathrm{p}<0.001)$ in water absorption capacity, oil absorption capacity, swelling power and per cent solubility of flour among the sorghum genotypes.

The water absorption capacity, oil absorption capacity, swelling power and per cent solubility of most of traditional sorghum landraces were on par with M35-1 and they ranged from 93.6 to $126.33 \mathrm{ml} / 100 \mathrm{~g}, 82.93$ to $119.8 \mathrm{ml} / 100 \mathrm{~g}, 6.37$ to $10.90 \mathrm{~g} / \mathrm{g}$ and 16.90 to 37.80 per cent. The different range of water and oil absorption capacity in different sorghum varieties were reported by Akajiaku, et al., 2017 (2.850 to $3.405 \mathrm{~g} / \mathrm{cm}^{3}$ and 1.320 to $\left.1.480 \mathrm{~g} / \mathrm{cm}^{3}\right)$, Rao et al., 2015 (7.50 to 9.29 and 1.855 to $1.977 \mathrm{~g} / \mathrm{g}$ ) and Thilagavathi et al., 2015 (74.08 to $76.83 \mathrm{ml} / 100 \mathrm{~g}$ and 73.58 to $85.57 \mathrm{ml} / 100 \mathrm{~g}$ ).

Proximate composition of sorghum genotypes is presented in table 3 . There was significant variation in moisture, crude fat, crude protein, ash, crude fiber and carbohydrate content among the sorghum genotypes $(\mathrm{P} \leq 0.001)$. The moisture, fat and carbohydrate content of traditional sorghum landraces were on par with M35-1 and they ranged from 10.76 to 12.47 per cent, 1.10 to 4.62 per cent and 64.28 to 72.27 per cent, respectively. The ash content of traditional sorghum landraces was lower compared to M35-1. This might be due to genetic variability and difference in mineral content.

The crude protein and crude fiber content in most of the traditional sorghum landraces were higher compared to M35-1 variety. The presence of total nitrogen content in the soil and minerals such as molybdenum and total chlorine tend to increase the grain protein content.

Higher crude fiber levels can be attributed to higher bran portion of grain. Different researchers reported various range for moisture, crude protein, fat, ash, fiber and carbohydrate content of sorghum genotypes. Prabhakar et al., 2016 showed the moisture, protein, fat, ash and total carbohydrate content in the Sorghum varieties were found in the range of 12 to 13.9 per cent, 9.8 to 15.08 per cent, 3.8 to 4.71 per cent, 1.89 to 2.06 per cent and 65.8 to 70.52 per cent, respectively. Tasie et al., 2020, moisture, ash, crude fat, crude fiber, crude protein, and $\mathrm{CHO}$ varied from 9.66 to $12.94,1.12$ to $2.29,2.48$ to $4.60,2.17$ to $8.59,8.20$ to 16.48 , and 67.56 to 76.42 , respectively. 
Table.1 Physical properties of sorghum genotypes

\begin{tabular}{|c|c|c|c|c|c|c|}
\hline \multirow[t]{2}{*}{ Sorghum genotypes } & \multirow{2}{*}{$\begin{array}{l}100 \text { kernel } \\
\text { weight }\end{array}$} & \multirow{2}{*}{$\begin{array}{l}100 \text { kernel } \\
\text { volume }\end{array}$} & \multirow[t]{2}{*}{ Bulk Density } & \multicolumn{3}{|c|}{ Colour } \\
\hline & & & & $\mathbf{L}^{*}$ & $\mathbf{a}^{*}$ & $\mathbf{b}^{*}$ \\
\hline Annigeri-1 & $3.05 \pm 0.12^{* \mathrm{gh}}$ & $3.67 \pm 0.12^{* a}$ & $0.83 \pm 0.04^{\mathrm{e}}$ & $85.03 \pm 0.12^{* \mathrm{f}}$ & $2.34 \pm 0.04^{* e f}$ & $9.15 \pm 0.03^{* j k}$ \\
\hline Basavanapada & $3.02 \pm 0.16^{* \mathrm{ghi}}$ & $2.13 \pm 0.12^{* \mathrm{hi}}$ & $1.42 \pm 0.07^{* a}$ & $86.22 \pm 0.14^{* d}$ & $2.18 \pm 0.02^{* \mathrm{~g}}$ & $9.01 \pm 0.09^{* \mathrm{kl}}$ \\
\hline Basavanamuti jola & $2.85 \pm 0.16^{h i j k}$ & $2.47 \pm 0.23^{\mathrm{fg}}$ & $1.16 \pm 0.05^{* b c d}$ & $84.03 \pm 0.09^{* h i}$ & $3.17 \pm 0.09^{* b}$ & $9.40 \pm 0.12^{* g h i}$ \\
\hline Bidar kudichandaki-1 & $2.64 \pm 0.05^{\mathrm{kl}}$ & $3.13 \pm 0.12^{b}$ & $0.83 \pm 0.04^{\mathrm{e}}$ & $85.77 \pm 0.19^{*}$ & $2.44 \pm 0.00^{* \mathrm{~cd}}$ & $10.56 \pm 0.19^{* a}$ \\
\hline Billigunda-local & $2.87 \pm 0.05^{\text {hijk }}$ & $2.47 \pm 0.12^{\text {fg }}$ & $1.16 \pm 0.05^{* b c d}$ & $84.90 \pm 0.05^{* f}$ & $3.20 \pm 0.09^{* b}$ & $9.18 \pm 0.14^{* i j k}$ \\
\hline Doddamaladandi & $3.54 \pm 0.08^{* \mathrm{~cd}}$ & $2.93 \pm 0.12^{b c d}$ & $1.21 \pm 0.02^{* \mathrm{bcd}}$ & $83.98 \pm 0.19^{* h i}$ & $2.32 \pm 0.07^{* \mathrm{f}}$ & $8.88 \pm 0.05^{* 1} \mathrm{~m}$ \\
\hline Giddamaldandi & $2.79 \pm 0.07^{1 \mathrm{jkl}}$ & $2.33 \pm 0.12^{* \mathrm{gh}}$ & $1.20 \pm 0.06^{* b c d}$ & $84.47 \pm 0.28^{* g}$ & $3.21 \pm 0.00^{* b}$ & $9.12 \pm 0.06^{* j \mathrm{jk}}$ \\
\hline Gundteni jola & $2.89 \pm 0.05^{* h i j}$ & $2.33 \pm 0.23^{\mathrm{gh}}$ & $1.24 \pm 0.13^{* b c}$ & $85.11 \pm 0.18^{* f}$ & $3.26 \pm 0.01^{* a b}$ & $9.82 \pm 0.02^{* c d}$ \\
\hline Ichangi-local-2 & $3.66 \pm 0.16^{* \mathrm{c}}$ & $3.00 \pm 0.00^{\mathrm{bc}}$ & $1.22 \pm 0.05^{* \mathrm{bcd}}$ & $85.59 \pm 0.00 * \mathrm{e}$ & $2.51 \pm 0.02^{* \mathrm{c}}$ & $9.66 \pm 0.011^{*}$ cdef \\
\hline Kantaavaradi & $3.12 \pm 0.12^{* \mathrm{fg}}$ & $2.67 \pm 0.30^{\mathrm{def}}$ & $1.18 \pm 0.15^{* b c d}$ & $83.65 \pm 0.04^{j}$ & $2.37 \pm 0.01^{* \operatorname{def}}$ & $9.14 \pm 0.05^{* j k}$ \\
\hline Kodamurki-local & $3.39 \pm 0.24^{* d e}$ & $3.13 \pm 0.12^{b}$ & $1.09 \pm 0.11^{\mathrm{d}}$ & $86.84 \pm 0.38^{* b}$ & $2.04 \pm 0.09^{* h}$ & $10.08 \pm 0.24^{* b}$ \\
\hline Lakamapur-local & $4.23 \pm 0.32^{* a}$ & $3.53 \pm 0.12^{* a}$ & $1.20 \pm 0.05^{* b c d}$ & $83.78 \pm 0.05^{* \mathrm{ij}}$ & $3.33 \pm 0.04^{* a}$ & $9.79 \pm 0.03^{* \text { cde }}$ \\
\hline Madabhavi- local & $3.11 \pm 0.01^{* \mathrm{fg}}$ & $2.53 \pm 0.12^{\mathrm{efg}}$ & $1.23 \pm 0.06^{* \mathrm{bcd}}$ & $84.24 \pm 0.11^{* \mathrm{gh}}$ & $2.43 \pm 0.10^{*} \mathrm{cde}$ & $9.57 \pm 0.09 *$ efgh \\
\hline Manadandagi jola & $3.43 \pm 0.10^{* d e}$ & $3.00 \pm 0.20^{\mathrm{bc}}$ & $1.14 \pm 0.04^{* b c d}$ & $85.20 \pm 0.20^{* \mathrm{f}}$ & $2.51 \pm 0.04^{* c}$ & $9.82 \pm 0.03^{* c d}$ \\
\hline Muddi hale jola & $2.78 \pm 0.10^{\mathrm{jkl}}$ & $2.47 \pm 0.12^{\mathrm{fg}}$ & $1.13 \pm 0.05^{* b c d}$ & $86.50 \pm 0.30 * \mathrm{~cd}$ & $1.96 \pm 0.09^{* \mathrm{~h}}$ & $9.60 \pm 0.40^{*}$ defg \\
\hline Narendra-local & $3.50 \pm 0.10^{* \mathrm{~cd}}$ & $2.80 \pm 0.20^{\text {cde }}$ & $1.25 \pm 0.06^{* b}$ & $86.63 \pm 0.02^{* b c}$ & $1.98 \pm 0.02^{* h}$ & $9.85 \pm 0.00^{* c}$ \\
\hline Nilugal & $2.64 \pm 0.03^{\mathrm{kl}}$ & $2.40 \pm 0.00^{* \mathrm{fgh}}$ & $1.10 \pm 0.01^{\mathrm{cd}}$ & $87.70 \pm 0.10^{* a}$ & $1.76 \pm 0.03^{* j}$ & $10.09 \pm 0.03^{* b}$ \\
\hline Rekkada jola & $3.28 \pm 0.04^{* e t}$ & $2.80 \pm 0.20^{*}$ cde & $1.17 \pm 0.08^{* \mathrm{bcd}}$ & $84.94 \pm 0.00^{* t}$ & $2.41 \pm 0.03^{* \mathrm{de}}$ & $9.20 \pm 0.07^{* i j k}$ \\
\hline SBABM jola & $4.01 \pm 0.05^{* b}$ & $3.20 \pm 0.00^{* b}$ & $1.25 \pm 0.02^{* b}$ & $84.99 \pm 0.29^{* \mathrm{f}}$ & $2.36 \pm 0.03^{* \text { def }}$ & $9.48 \pm 0.10^{* \text { *gh }}$ \\
\hline Yenigararati & $2.31 \pm 0.07 \mathrm{~m}$ & $2.00 \pm 0.20^{* 1}$ & $1.16 \pm 0.15^{* b c d}$ & $84.21 \pm 0.08^{* \mathrm{gh}}$ & $2.00 \pm 0.00^{* h}$ & $9.34 \pm 0.04^{* h i j}$ \\
\hline M35-1 & $2.57 \pm 0.05^{1}$ & $2.80 \pm 0.20^{\text {cde }}$ & $0.83 \pm 0.04^{\mathrm{e}}$ & $83.36 \pm 0.07^{\mathrm{k}}$ & $1.54 \pm 0.03^{\mathrm{i}}$ & $8.76 \pm 0.10 \mathrm{~m}$ \\
\hline Mean \pm SD & $3.13 \pm 0.49$ & $2.75 \pm 0.45$ & $1.15 \pm 0.15$ & $85.10 \pm 1.16$ & $2.44 \pm 0.51$ & $9.50 \pm 0.46$ \\
\hline F-value & $45.018 * *$ & $22.582 * *$ & $9.668 * *$ & $138.811 * *$ & $297.179 * *$ & $37.323 * *$ \\
\hline SEM \pm & 0.23 & 0.29 & 0.04 & 0.32 & $\mathbf{0 . 0 3}$ & 0.23 \\
\hline CD at $1 \%$ & 0.66 & 0.82 & 0.13 & 0.9 & 0.09 & 0.66 \\
\hline
\end{tabular}

Means followed by asterisk (*) differed from the control group by the Dunnett test $(\mathrm{p}<0.05)$.

Means followed by different letters in the same column are statistically different by the Duncan test $(\mathrm{p}<0.05)$ 
Table.2 Functional properties of sorghum genotypes

\begin{tabular}{|c|c|c|c|c|}
\hline Sorghum landraces & $\begin{array}{l}\text { Water absorbtion } \\
\text { capacity }(\%)\end{array}$ & $\begin{array}{l}\text { Oil absorbtion capacity } \\
(\%)\end{array}$ & Swelling power (g/g) & Solubility (\%) \\
\hline Annigeri-1 & $112 \pm 0.03^{\text {abcdef }}$ & $90 \pm 0.00^{\text {bcde }}$ & $9.52 \pm 0.06^{\mathrm{abc}}$ & $17.70 \pm 6.83^{\mathrm{g}}$ \\
\hline Basavanapada & $110 \pm 0.03^{\text {bcdef }}$ & $85 \pm 0.02^{\mathrm{de}}$ & $7.90 \pm 0.49^{\mathrm{de}}$ & $30.90 \pm 1.26^{\mathrm{abcd}}$ \\
\hline Basavanamuti jola & $112 \pm 0.09^{\text {abcdef }}$ & $83 \pm 0.00^{\mathrm{de}}$ & $8.73 \pm 0.07^{\mathrm{cd}}$ & $30.10 \pm 6.98^{\text {abcde }}$ \\
\hline Bidar kudichandaki-1 & $111 \pm 0.01^{\text {abcdef }}$ & $94 \pm 0.01^{\mathrm{bcd}}$ & $8.54 \pm 0.61^{\mathrm{cde}}$ & $16.90 \pm 5.77^{\mathrm{g}}$ \\
\hline Billigunda-local & $94 \pm 0.05^{\mathrm{g}}$ & $89 \pm 0.01^{\text {cde }}$ & $10.30 \pm 0.9^{\mathrm{ab}}$ & $36.30 \pm 2.33^{* a b}$ \\
\hline Doddamaladandi & $107 \pm 0.09^{\operatorname{detg}}$ & $96 \pm 0.00^{\mathrm{bcd}}$ & $6.37 \pm 2.39^{* t}$ & $31.80 \pm 1.57^{* a b c d}$ \\
\hline Giddamaldandi & $111 \pm 0.00^{\text {bcdef }}$ & $95 \pm 0.03^{\mathrm{bcd}}$ & $10.00 \pm 0.21^{\mathrm{abc}}$ & $27.80 \pm 4.31^{\text {cdef }}$ \\
\hline Gundteni jola & $108 \pm 0.00^{\text {cdetg }}$ & $76 \pm 0.00^{\mathrm{e}}$ & $10.90 \pm 0.30^{\mathrm{a}}$ & $37.40 \pm 1.05^{\mathrm{a}}$ \\
\hline Ichangi-local-2 & $115 \pm 0.00^{\mathrm{abcde}}$ & $105 \pm 0.13^{b}$ & $9.05 \pm 0.12^{\text {bcd }}$ & $31.50 \pm 1.27^{\mathrm{abcd}}$ \\
\hline Kantaavaradi & $107 \pm 0.12^{\text {cdefg }}$ & $98 \pm 0.12^{b c d}$ & $8.46 \pm 0.34^{\text {cde }}$ & $37.80 \pm 5.06^{* a}$ \\
\hline Kodamurki-local & $121 \pm 0.01^{\mathrm{abcd}}$ & $92 \pm 0.01^{\text {bcde }}$ & $8.49 \pm 0.09^{\text {cde }}$ & $28.30 \pm 1.35^{\text {bcdef }}$ \\
\hline Lakamapur-local & $110 \pm 0.08^{\text {bcdef }}$ & $120 \pm 0.30^{* a}$ & $8.42 \pm 0.10^{\text {cde }}$ & $23.10 \pm 3.85^{\text {efg }}$ \\
\hline Madabhavi- local & $101 \pm 0.01^{\mathrm{efg}}$ & $87 \pm 0.02^{\text {cde }}$ & $7.09 \pm 0.82^{*}$ & $26.30 \pm 1.83^{\text {def }}$ \\
\hline Manadandagi jola & $124 \pm 0.01^{\mathrm{ab}}$ & $84 \pm 0.00^{\mathrm{de}}$ & $8.86 \pm 2.22^{b c d}$ & $20.80 \pm 1.89^{f g}$ \\
\hline Muddi hale jola & $126 \pm 0.00^{\mathrm{a}}$ & $88 \pm 0.01^{\text {cde }}$ & $8.72 \pm 0.03^{\mathrm{cd}}$ & $36.00 \pm 0.32^{\mathrm{ab}}$ \\
\hline Narendra-local & $122 \pm 0.21^{a b c}$ & $89 \pm 0.01^{\text {cde }}$ & $9.26 \pm 0.00^{\mathrm{bcd}}$ & $32.10 \pm 1.89^{* a b c d}$ \\
\hline Nilugal & $114 \pm 0.00^{\text {abcde }}$ & $93 \pm 0.00^{\mathrm{bcd}}$ & $9.20 \pm 0.44^{\text {bcd }}$ & $29.20 \pm 1.79^{\text {bcde }}$ \\
\hline Rekkada jola & $113 \pm 0.05^{\mathrm{abcde}}$ & $83 \pm 0.02^{\mathrm{de}}$ & $8.96 \pm 0.08^{b c d}$ & $32.10 \pm 0.00^{* a b c d}$ \\
\hline SBABM jola & $97 \pm 0.20^{\operatorname{tg}}$ & $82 \pm 0.01^{\mathrm{de}}$ & $9.35 \pm 0.17^{\text {bcd }}$ & $32.10 \pm 0.00^{\text {abcd }}$ \\
\hline Yenigararati & $108 \pm 0.02^{\text {cdefg }}$ & $103 \pm 0.07^{b c}$ & $8.43 \pm 0.32^{\text {cde }}$ & $35.90 \pm 7.28^{* a b c}$ \\
\hline M35-1 & $113 \pm 0.01^{\text {abcde }}$ & $92 \pm 0.03^{b c d}$ & $9.64 \pm 0.30^{a b c}$ & $21.40 \pm 9.63^{f g}$ \\
\hline Mean \pm SD & $111 \pm 0.1$ & $92 \pm 0.11$ & $8.87 \pm 1.19$ & 29.35 \pm 7.09 \\
\hline F-value & 3.331** & $4.264 * *$ & 4.729** & $6.703 * *$ \\
\hline SEM \pm & 0.04 & 0.04 & 0.46 & 0.42 \\
\hline CD at $1 \%$ & 0.13 & 0.13 & 1.32 & 1.21 \\
\hline
\end{tabular}

Means followed by asterisk (*) differed from the control group by the Dunnett test $(\mathrm{p}<0.05)$.

Means followed by different letters in the same column are statistically different by the Duncan test $(\mathrm{p}<0.05)$ 
Table.3 Proximate composition of sorghum genotypes (\%)

\begin{tabular}{|c|c|c|c|c|c|c|}
\hline Sorghum landraces & Moisture & Protein & Fat & Ash & Crude fiber & $\begin{array}{c}\text { Total } \\
\text { Carbohydrate }\end{array}$ \\
\hline Annigeri-1 & $10.84 \pm 0.03^{\mathrm{gh}}$ & $13.02 \pm 0.08^{* \mathrm{~cd}}$ & $3.42 \pm 0.37^{* a b c d}$ & $1.41 \pm 0.04^{* \text { ghij }}$ & $3.01 \pm 0.80 * \mathrm{ef}$ & $68.30 \pm 0.59^{\text {def }}$ \\
\hline Basavanapada & $11.24 \pm 0.43^{\text {cdef }}$ & $12.93 \pm 0.01^{* \mathrm{~cd}}$ & $1.10 \pm 0.75^{\mathrm{i}}$ & $1.60 \pm 0.03^{\text {cdef }}$ & $2.71 \pm 0.88^{* \mathrm{fg}}$ & $70.42 \pm 0.53^{b c}$ \\
\hline Basavanamuti jola & $12.47 \pm 0.17^{* a}$ & $11.39 \pm 0.16^{* \mathrm{ghi}}$ & $1.72 \pm 0.27^{\mathrm{fghi}}$ & $1.58 \pm 0.06^{\mathrm{def}}$ & $6.99 \pm 0.20^{* a}$ & $65.86 \pm 0.14^{* g h}$ \\
\hline Bidar kudichandaki-1 & $11.15 \pm 0.25^{\mathrm{bcdefg}}$ & $11.89 \pm 0.00^{* e f g}$ & $2.50 \pm 1.10^{\text {cdetgh }}$ & $1.50 \pm 0.01^{* \mathrm{fgh}}$ & $1.53 \pm 0.25^{* \mathrm{~h}}$ & $71.44 \pm 1.11^{\mathrm{ab}}$ \\
\hline Billigunda-local & $11.33 \pm 0.17^{\mathrm{cd}}$ & $11.62 \pm 1.12^{* \mathrm{fgh}}$ & $1.80 \pm 0.25^{\mathrm{fghi}}$ & $1.72 \pm 0.02^{\mathrm{bc}}$ & $4.00 \pm 0.43^{\mathrm{cd}}$ & $69.54 \pm 0.60^{c d}$ \\
\hline Doddamaladandi & $11.34 \pm 0.43^{\mathrm{cd}}$ & $12.58 \pm 0.16^{* \mathrm{cde}}$ & $3.37 \pm 1.62^{* \text { abcde }}$ & $1.63 \pm 0.03^{\mathrm{cde}}$ & $6.81 \pm 0.05^{* a}$ & $64.28 \pm 1.86^{* i}$ \\
\hline Giddamaldandi & $12.30 \pm 0.12^{* a}$ & $13.17 \pm 0.13^{* \mathrm{c}}$ & $1.46 \pm 0.07^{\mathrm{ghi}}$ & $1.51 \pm 0.07^{*} \mathrm{efg}$ & $2.77 \pm 0.26^{* \mathrm{fg}}$ & $68.79 \pm 0.12^{\text {de }}$ \\
\hline Gundteni jola & $12.31 \pm 0.14^{* a}$ & $11.48 \pm 0.33^{* \mathrm{gh}}$ & $1.89 \pm 0.26^{\text {tghi }}$ & $1.28 \pm 0.00^{* \mathrm{kI}}$ & $1.93 \pm 0.29^{* g h}$ & $71.11 \pm 0.23^{\mathrm{abc}}$ \\
\hline Ichangi-local-2 & $11.46 \pm 0.16^{\mathrm{c}}$ & $11.44 \pm 0.26^{* g h i}$ & $2.77 \pm 1.42^{\text {bcdefg }}$ & $1.30 \pm 0.18^{* j \mathrm{kl}}$ & $2.57 \pm 0.79^{* \mathrm{fg}}$ & $70.47 \pm 1.92^{\mathrm{bc}}$ \\
\hline Kantaavaradi & $10.93 \pm 0.38^{\text {fgh }}$ & $12.47 \pm 0.09^{*}$ cdef & $1.67 \pm 0.08^{\text {fghi }}$ & $1.67 \pm 0.04^{\mathrm{bcd}}$ & $2.21 \pm 0.31^{* \mathrm{fgh}}$ & $71.04 \pm 0.65^{\mathrm{abc}}$ \\
\hline Kodamurki-local & $11.30 \pm 0.24^{\text {cde }}$ & $14.75 \pm 1.48^{* a}$ & $2.10 \pm 0.45^{\text {efghi }}$ & $1.43 \pm 0.07^{* \mathrm{ghi}}$ & $3.65 \pm 0.42^{\mathrm{de}}$ & $66.77 \pm 0.30^{* \mathrm{fg}}$ \\
\hline Lakamapur-local & $11.37 \pm 0.00^{\mathrm{cd}}$ & $13.37 \pm 0.08^{* b c}$ & $3.52 \pm 0.13^{* a b c}$ & $1.96 \pm 0.00^{* a}$ & $4.95 \pm 0.98^{b}$ & $64.83 \pm 0.78^{* h i}$ \\
\hline Madabhavi- local & $12.10 \pm 0.00^{* a b}$ & $10.60 \pm 0.10^{\mathrm{ij}}$ & $2.80 \pm 0.50^{\text {bcdef }}$ & $1.78 \pm 0.00^{b}$ & $2.20 \pm 0.30^{* \operatorname{fgh}}$ & $70.50 \pm 0.00^{b c}$ \\
\hline Manadandagi jola & $11.93 \pm 0.12^{* b}$ & $13.30 \pm 0.65^{* c}$ & $1.46 \pm 0.12^{\mathrm{ghi}}$ & $1.38 \pm 0.14^{* h i j k}$ & $2.33 \pm 0.10^{* \mathrm{fgh}}$ & $69.60 \pm 0.65^{\mathrm{cd}}$ \\
\hline Muddi hale jola & $11.83 \pm 0.10^{* \mathrm{~b}}$ & $10.21 \pm 0.01^{\mathrm{j}}$ & $1.64 \pm 0.00^{\text {fghi }}$ & $1.26 \pm 0.03^{* \mathrm{kl}}$ & $2.79 \pm 0.14^{* \mathrm{fg}}$ & $72.27 \pm 0.01^{\mathrm{a}}$ \\
\hline Narendra-local & $11.06 \pm 0.06^{\text {defgh }}$ & $14.15 \pm 0.69^{* a b}$ & $2.15 \pm 0.45^{\operatorname{defghi}}$ & $2.00 \pm 0.00^{* a}$ & $2.35 \pm 0.42^{* \operatorname{fgh}}$ & $68.29 \pm 1.62^{* \text { def }}$ \\
\hline Nilugal & $10.76 \pm 0.05^{* h}$ & $12.84 \pm 0.10^{* \mathrm{~cd}}$ & $4.62 \pm 0.22^{* a}$ & $1.33 \pm 0.03^{* \mathrm{ijkl}}$ & $2.96 \pm 0.07^{* e f}$ & $67.49 \pm 0.24^{* e f}$ \\
\hline Rekkada jola & $12.42 \pm 0.18^{* a}$ & $10.74 \pm 0.24^{h i j}$ & $1.22 \pm 0.43^{\mathrm{hi}}$ & $1.22 \pm 0.00^{* 1}$ & $2.85 \pm 0.46^{* e f}$ & $71.55 \pm 0.39^{\mathrm{ab}}$ \\
\hline SBABM jola & $12.32 \pm 0.08^{* a}$ & $10.89 \pm 0.17^{\mathrm{hij}}$ & $1.67 \pm 0.08^{\text {tghi }}$ & $1.24 \pm 0.02^{* 1}$ & $4.32 \pm 0.40^{\mathrm{bcd}}$ & $69.56 \pm 0.37^{\mathrm{cd}}$ \\
\hline Yenigararati & $10.94 \pm 0.02^{\text {efgh }}$ & $12.23 \pm 0.16^{* \operatorname{defg}}$ & $3.94 \pm 1.55^{* a b}$ & $1.27 \pm 0.15^{* \mathrm{kl}}$ & $3.96 \pm 0.24^{\mathrm{cd}}$ & $67.66 \pm 1.31^{\mathrm{ef}}$ \\
\hline M35-1 & $11.30 \pm 0.07^{\text {cde }}$ & $10.12 \pm 0.36^{j}$ & $1.45 \pm 0.20^{\mathrm{ghi}}$ & $1.71 \pm 0.07^{b c}$ & $4.69 \pm 0.03^{b c}$ & $70.73 \pm 0.12^{\mathrm{abc}}$ \\
\hline Mean \pm SD & $11.56 \pm 0.60$ & $1.51 \pm 0.24$ & $2.30 \pm 1.12$ & $12.15 \pm 1.32$ & $3.41 \pm 1.50$ & $69.07 \pm 2.33$ \\
\hline F-value & $25.350 * *$ & $33.414 * *$ & $6.040 * *$ & $21.120 * *$ & $30.698 * *$ & $20.426 * *$ \\
\hline SEm \pm & 0.11 & 0.04 & 0.4 & 0.28 & 0.27 & 0.5 \\
\hline CD & 0.32 & 0.02 & 1.14 & 0.79 & 0.76 & 1.42 \\
\hline
\end{tabular}

Means followed by asterisk (*) differed from the control group by the Dunnett test $(\mathrm{p}<0.05)$.

Means followed by different letters in the same column are statistically different by the Duncan test $(\mathrm{p}<0.05)$ 
It can be concluded that the most of the landraces had significant higher 100 kernel weight, bulk density, colour of grains $\left(\mathrm{L}, \mathrm{a}^{*}\right.$ and $b^{*}$ ), crude protein and crude fiber content than M35-1. The 100 kernel volume, water absorption capacity, oil absorption capacity, swelling power and per cent solubility of more than half of the sorghum landraces were on par with M35-1. Whereas the ash content ranged from 1.22 to 2.00 per cent and was significantly lower compared to M35-1.

\section{References}

Akajiaku, L. O., Nwosu, J. N., Kabuo, N. O., Odimegwu, E. N., Umelo, M. C., Unegbu, V. C. 2017.Using sorghum flour as part substitute of wheat flour in noodles making. MOJ Food Processing and Technology. 5(2): 250.

Anonymous. 2005. Official Methods of Analysis, Association of Official Analytical Chemists, 18th edition, Washington, DC, USA.

Itagi, C. and Hemalatha, S. 2017.Variability in grain physico-chemical composition in different sorghum [Sorghum bicolor] genotypes. International Journal of Current Microbiology and Applied Sciences. 6(7): 2610-2618.

Patekar, S. D., More, D. R. and Hashmi, S. I., 2017, Studies on physico-chemical properties and minerals content from different sorghum genotypes. Journal of Pharmacognosy

and Phytochemistry.6(5): 600-604.

Prabhakar, B., Sanganamoni, M., Shivashankar, S. and Babu, N., 2016, Studied on physico-chemical analysis of sorghum varieties. Int. J. Agric. Sci. Res., 6(1): $87-92$.

\section{How to cite this article:}

Preeti Ramakant Hungund, Usha Malagi and Hanamaratti, N. G. 2021. Physico-Chemical and Functional Properties of Traditional Sorghum Landraces. Int.J.Curr.Microbiol.App.Sci. 10(04): 559-567. doi: https://doi.org/10.20546/ijcmas.2021.1004.055
Quin, J. R. and Paton, D. A. 1983.Practical Measurement of Water Hydration Capacity of Protein Materials.Cereal Chemistry. 56: 38-44.

Rao, B. D., Anis, A. M., Kalpana, K., Sunooj, K. V, Patil, J. V. and Ganesh, T. 2015. Influence of milling methods and particle size on hydration properties of sorghum flour and quality of sorghum biscuits.LWT - Food Science and Technology. 67: 8-13.

Schoch, T. J., 1964, Swelling Power and Solubility of Granular Starches. In: Carbohydrate Chemistry. Ed. Whistler, R. L., Smith, R. J., Miller, N. J. and Wolform, M. I., Academic Press, New York, pp. 106-108.

Sosulski, F. W., Garatt, M. O. and Slinkard, A. E., 1976, Functional properties of ten legume flours. International Journal of Food Science and Technology 9: 66-69.

Tasie, M. M. and Gebreyes, B. G. 2020.Characterization of nutritional, antinutritional, and mineral contents of thirty-five sorghum varieties grown in Ethiopia. International Journal of Food Science.

https://doi.org/10.1155/2020/8243617.

Thilagavathi, T., Kanchana, S., Banumathi, P., Hemalatha, G., Vanniarajani, C., Sundar, M. and Ilamaran, M. 2015.Physicochemical and functional characteristics of selected millets and pulses. Indian journal of science and technology. 8(7): 147-155.

Yang, L., Browning, J. D. and Awika, J.M. 2009. Sorghum 3-deoxyanthocyanins possess strong phase II enzyme inducer activity and cancer cell growth inhibition properties. Journal of Agriculture and Food Chemistry.57:1797-1804. 